\title{
Role of aggregated medin in the pathogenesis of thoracic aortic aneurysm and dissection
}

\author{
Siwei Peng ${ }^{1, *}$, Annika Larsson ${ }^{1, *}$, Erik Wassberg ${ }^{2}$, Pär Gerwins ${ }^{1,3}$, Stefan Thelin ${ }^{2}$, Xin Fu $^{4}$ and Per Westermark ${ }^{1}$
}

The pathogenesis of sporadic thoracic aortic aneurysm and dissection, which may lead to rupture of the aorta, remains largely unknown. Amyloid deposits, formed from the medin peptide, are very prevalent in the media of the thoracic aorta. We have studied the occurrence of medin-derived amyloid in specimens from patients with thoracic aortic aneurysm, aortic dissection type A and normal dimensioned aorta. Surprisingly, the amount of amyloid was significantly lower in the aneurysm and dissection groups $\left(0.63 \pm 0.13\right.$ and $0.36 \pm 0.24$ amyloid particles per $\mathrm{mm}^{2}$, respectively) compared to the control material $(2.37 \pm 0.58)$. However, focal medin immunoreactivity not associated with amyloid was found more conspicuously in the media of the two diseased groups. Recent amyloid research indicates that prefibrillar oligomeric aggregates, rather than mature amyloid fibrils, are toxic to the surrounding cells. The non-amyloid medin immunoreactivity observed may represent such toxic oligomers. This is supported by the fact that aggregated medin induced death of aortic smooth muscle cells in vitro. In addition, cells incubated together with medin increased the production of matrix metalloproteinase-2, a protease that degrades elastin and collagen and subsequently weakens the vessel wall. We therefore propose that medin oligomers are involved in the degeneration process of sporadic thoracic aortic aneurysm and dissection.

Laboratory Investigation (2007) 87, 1195-1205; doi:10.1038/labinvest.3700679; published online 1 October 2007

KEYWORDS: amyloid; aneurysm; dissection; lactadherin; medin; thoracic aorta

Thoracic aortic dissection and thoracic aortic aneurysm are life-threatening conditions associated with degenerative changes in the media. In rare instances, the disorders are associated with Marfan syndrome in which missense mutations in the fibrillin-1 gene lead to defective elastic tissue. However, in most cases, the pathogenesis of these conditions is poorly understood. Degeneration of elastic structures and extracellular deposits of glycosaminoglycan-rich material, the latter often referred to as cystic medial necrosis, are typical histopathologic findings. ${ }^{1,2}$ Increased production or presence of certain matrix metalloproteinases (MMPs), particularly MMP-1, MMP-2 and MMP-9, has been found in both thoracic aortic aneurysms and dissections. ${ }^{3-6}$ The resulting proteolysis leads to weakening of the aortic wall and appears to be a direct cause of the lesions. Why there is an upregulation of MMPs is unknown.

Small localized amyloid deposits are particularly common in the cardiovascular system. Amyloid in any location is characterized by its fibrillar ultrastructure, X-ray diffraction pattern and staining properties with Congo red and Thioflavin $\mathrm{T}$, characteristics that are all related to the high degree of $\beta$-pleated sheet content of amyloid. The most prevalent site of amyloid deposition in humans is the media of arteries, ${ }^{7-9}$ and these amyloid deposits have been found to be biochemically distinct from other cardiovascular amyloid forms. ${ }^{9,10}$ The main amyloid fibril protein is medin (or AMed), which is a 50 amino-acid residue internal cleavage product of the precursor protein lactadherin. ${ }^{11}$ Lactadherin is a 364 amino-acid residue protein found in many tissues ${ }^{11-14}$ and has many proposed functions. ${ }^{13,15-17}$ To these functions we have added the ability of lactadherin to bind to elastin, and we have suggested that lactadherin acts as a linker between smooth muscle cells and elastin through its RGD-sequence at the $\mathrm{N}$-terminal part and its medin part close to the $\mathrm{C}$ terminus. ${ }^{18}$

In Alzheimer's disease, type II diabetes and some other disorders amyloid is a histopathological hallmark (for review

\footnotetext{
${ }^{1}$ Department of Genetics and Pathology, Uppsala University, Uppsala, Sweden; ${ }^{2}$ Department of Surgical Sciences, Uppsala University, Uppsala, Sweden; ${ }^{3}$ Department of Oncology, Radiology and Clinical Immunology, Section for Radiology, Uppsala University, Uppsala, Sweden and ${ }^{4}$ Department of Women's and Children's Health, Uppsala University, Uppsala, Sweden

Correspondence: Dr P Westermark, MD, PhD, Rudbeck Laboratory, Department of Genetics and Pathology, Uppsala University, C5, SE-751 85 Uppsala, Sweden. E-mail: Per.Westermark@genpat.uu.se

*These authors contributed equally to this work.

Received 27 June 2007; revised 28 August 2007; accepted 28 August 2007
} 
see Merlini and Bellotti ${ }^{19}$ and Westermark ${ }^{20}$ ). After being overlooked for a long period of time, the potential importance of amyloid in the pathogenesis of these diseases has more recently come into focus. Exactly how aggregated proteins lead to tissue injury is not completely understood. Although amyloid deposits are space occupying and in this way may disturb normal function, recent data indicate that other mechanisms are more important. The fully developed amyloid fibrils may not be the most deleterious. Rather, smaller prefibrillar aggregates, often referred to as oligomers or protofibrils, have been shown to exert toxic effects on cells. ${ }^{21,22}$ Protein oligomers are believed to be important in the specific loss of neurons in Alzheimer's disease and of $\beta$ cells in type II diabetes. The oligomers bind to cells and, through mechanisms that are yet poorly understood, may lead to cell death. The formation of abnormal pores and pathological ion channels, increased oxidative stress, and interaction with local receptors (such as the receptor for advanced glycation end products) are events that have all been implied to be mediated by such protein aggregates. ${ }^{23-26}$

It is well known from other forms of amyloidosis that the involvement of arteries may induce medial degeneration and weakening of the vascular wall, leading to an increased risk for rupture. This can be seen in the systemic amyloidoses, but particularly in forms of intracranial amyloidosis, including $\mathrm{A} \beta$-amyloid. ${ }^{27,28}$ It has been shown in a mouse model of $\mathrm{A} \beta$ cerebral amyloid angiopathy that the amyloid deposits are associated with disorganized medial smooth muscle cells and a substantial loss of these cells. ${ }^{29,30}$ Therefore, it is logical to believe that the sometimes widely spread medin amyloid deposits in the thoracic aorta are associated with aortic wall weakening. In this study, we have studied the prevalence of medin in aortic tissues of patients suffering from sporadic thoracic aortic dissection and aneurysm. In addition, we studied the effect of medin in a smooth muscle cell culture system.

\section{MATERIALS AND METHODS Aortic Specimens}

Aortic specimens from 27 patients with thoracic aortic aneurysm (Table 1) and 10 with aortic dissection type A (Table 2) were obtained from patients undergoing elective or acute surgery. All tissue specimens were from the thoracic ascending aorta. Materials from three individuals with Marfan syndrome and two individuals with giant cell aortitis were excluded from the analysis owing to the specific pathogeneses of these diseases. For comparison, tissue specimens from the same region of the aorta were taken from 29 patients undergoing coronary artery bypass surgery (punch biopsies) or aortic valve-replacement surgery (Table 3). No obvious differences in risk factors between the three groups were observed. The control group showed no signs of medial weakness such as aortic dilation, that is, the thoracic ascending aortas had normal dimensions. The majority of individuals in all groups were males. The mean age in the control group was slightly higher than in the aneurysm group (68.0 vs 64.2 years). The mean age in the dissection group was 60.8 years. In all instances, the specimens were kept on ice during transport to the laboratory, where they were divided into a part that was fixed in buffered neutral $4 \%$ formaldehyde solution and embedded in paraffin and a remaining part that was kept at $-20^{\circ} \mathrm{C}$.

The Ethics Committee of Uppsala University Hospital approved all studies. Data were encoded to ensure patient protection and informed consent was obtained. All measurements were performed without knowledge about the nature of the material.

\section{Histological Methods}

Sections were stained with Congo red for demonstration of amyloid. ${ }^{31}$ Other sections were stained with a combination of aldehyde fuchsine (for elastin), van Gieson stain (for smooth muscle cells and collagen) and alcian blue (for glycosaminoglycans). Some sections, after treatment with these latter combined stains, were also treated for $10 \mathrm{~min}$ with Congo red B solution ${ }^{32}$ for simultaneous demonstration of different tissue components and amyloid.

\section{Immunohistochemical Methods}

Rabbit antiserum A179 was raised against a synthetic peptide corresponding to amino acid 245-256 (within the medin sequence) of lactadherin, as described. ${ }^{18,33}$ In Western blots, this antiserum detects lactadherin and medin but appears to be a medin-specific antibody in immunohistochemistry. It labels amyloid but not elastin-associated lactadherin in aortic and skin material. This immunoreaction is extinguished by pre-incubating the antiserum with its corresponding peptide. Immunohistochemistry using A179, diluted 1:4000, was performed on $5 \mu \mathrm{m}$ thick deparaffinized sections as described. ${ }^{18}$ For double staining with Congo red, immunolabeled sections were treated with Congo red B solution for $10 \mathrm{~min}$ and then mounted. Confocal microscopy was used for detailed analysis of the topographical relationship between amyloid and different tissue components. For this latter procedure, deparaffinized sections were incubated overnight with A179 (1:4000) and treated as described. ${ }^{18}$

\section{Quantitative Estimation of Amyloid Deposits and Immunolabeled Areas}

As the amyloid deposits in the aortic media are dispersed as very small aggregates and are sometimes very indistinctively demarcated and only weakly stained with Congo red, a semiquantitative method was used. The individual amyloid deposits in sections stained with Congo red were counted, and the area of the tissue was determined with the aid of a Leica Qwin Standard Y2.8 program (Leica Microsystems Imaging Solutions, Cambridge, UK). The amount of amyloid was then expressed as the number of deposits per $\mathrm{mm}^{2}$. Areas immunolabeled with antiserum A179 were also determined with the use of the Leica Qwin Standard Y2.8 program and 
Table 1 Aneurysm specimens

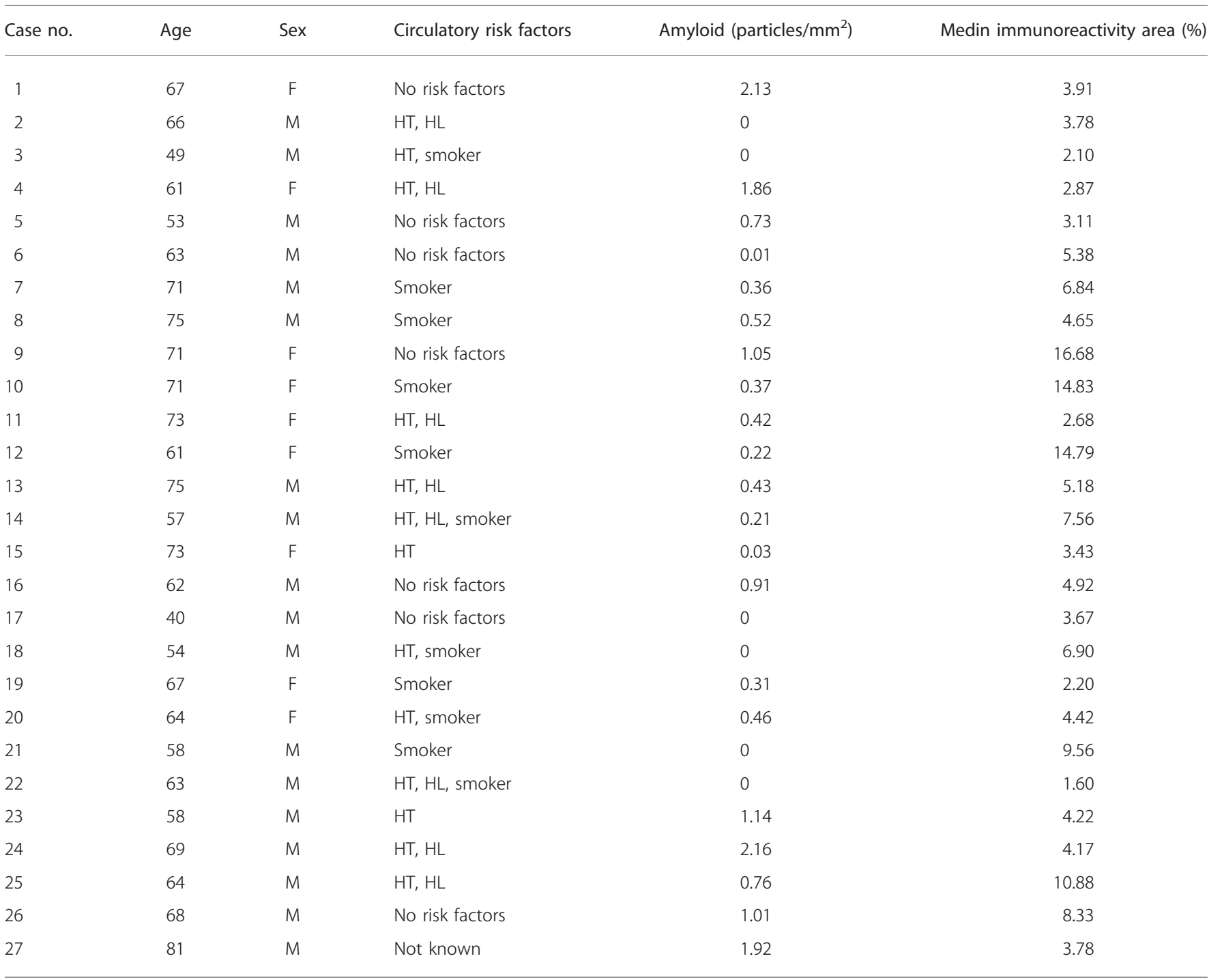

$F$, female; $H L$, hyperlipidemia; $H T$, hypertension; $M$, male.

Table 2 Dissection specimens

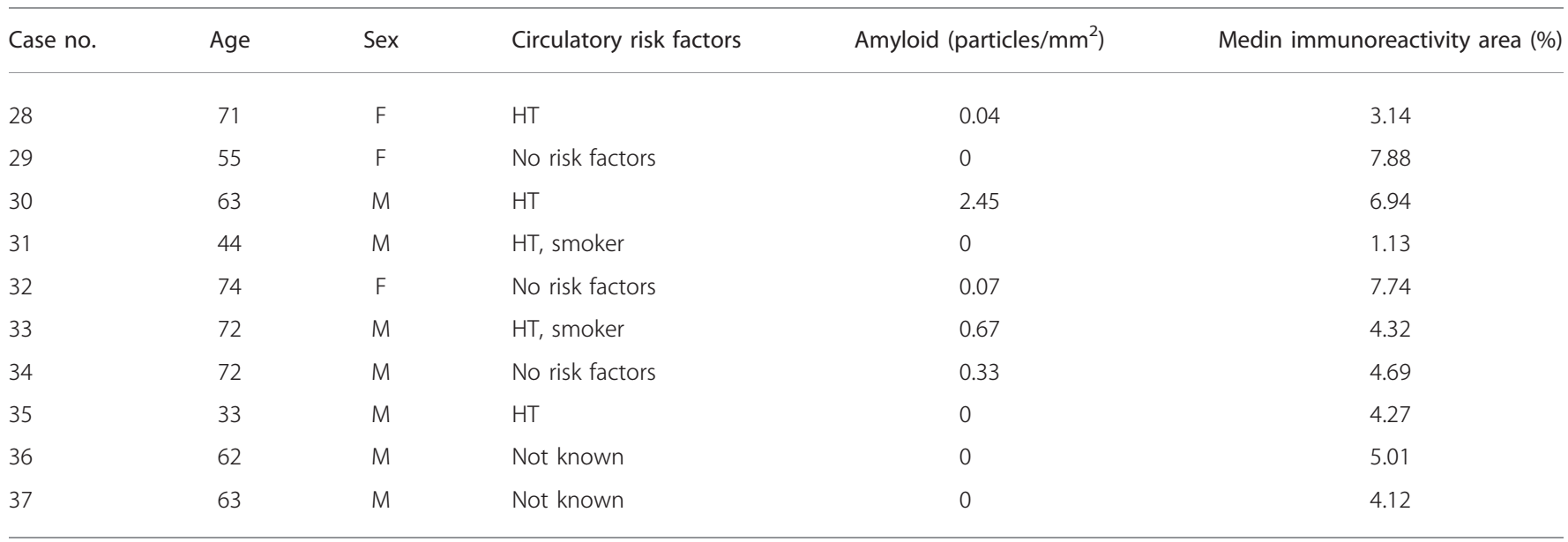

$F$, female; $H L$, hyperlipidemia; $H T$, hypertension; $M$, male. 
Table 3 Control specimens

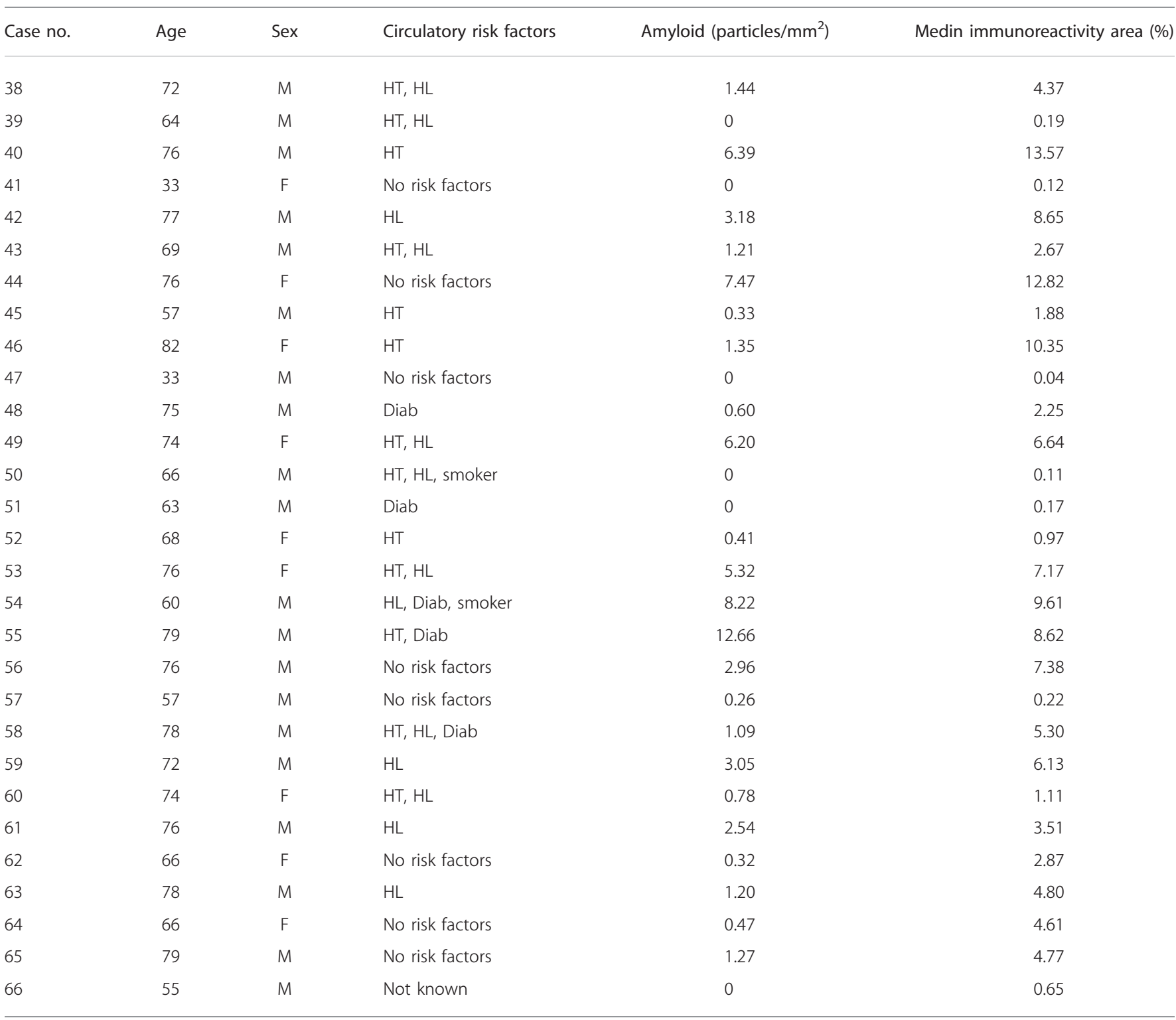

Diab, diabetes; $F$, female; $H L$, hyperlipidemia; $H T$, hypertension; $M$, male.

the results expressed as the percent of tissue area showing immunoreactivity.

\section{Protein Extraction}

Medial samples were homogenized on ice in $0.15 \mathrm{~mol} / \mathrm{l} \mathrm{NaCl}$ containing $0.05 \mathrm{~mol} / 1$ dithiothreitol and protease inhibitors $(0.2 \mathrm{mmol} / \mathrm{l}$ phenylmethylsulfone fluoride, $2 \mu \mathrm{mol} / \mathrm{l}$ leupeptin, $2 \mu \mathrm{mol} / 1$ pepstatin, $1 \mathrm{mmol} / 1 \varepsilon$-aminocaproic acid) and centrifuged $(27000 \mathrm{~g}, 30 \mathrm{~min})$. This was repeated four times. By this procedure, the amyloid fibrils end up in the pellet material, whereas the supernatants contain the watersoluble material. The supernatants were dialyzed against distilled water and lyophilized. Pellet material was washed in distilled water to remove salt and lyophilized. To solubilize amyloid, pellet material was treated with $6 \mathrm{~mol} / \mathrm{l}$ guanidine$\mathrm{HCl}$ in $0.1 \mathrm{~mol} / \mathrm{l}$ Tris $\mathrm{HCl}, \mathrm{pH} 8.0$, containing $0.1 \mathrm{~mol} / \mathrm{l}$ dithiothreitol and $0.2 \mathrm{~mol} / \mathrm{l}$ ethylenediaminetetraacetic acid overnight at room temperature. After centrifugation, the solution was dialyzed against distilled water and lyophilized.

\section{Cell Culture and Toxicity Studies}

Primary human smooth muscle cells of aortic origin from Clonetics (Cambrex, Walkersville, MD, USA) were grown in 96-well microtiter plates according to the recommendations of the distributor. On the day before medin treatment, the serum concentration in the culture medium was reduced from 5 to $0.5 \%$. Synthetically produced medin (Keck Biotechnology Resource Laboratory, New Haven, CT, USA) was 
treated as described ${ }^{18}$ to dissolve preformed aggregates. It was then dissolved in dimethylsulfoxide and diluted 1:10 with water to a peptide concentration of $0.5 \mathrm{mmol} / \mathrm{l}$. The medin solution was added either immediately to the cells or incubated at room temperature for various time periods before the toxicity study to obtain different aggregation states. Medin-derived amyloid-like fibrils (confirmed with Congo red staining of droplets on glass slides) were seen from day 5 . A fibrillar medin preparation was used in addition to medin that was freshly dissolved and not preincubated. The freshly dissolved medin solution mainly consists of monomers, but up to octamers were detected at size exclusion chromatography (Larsson et al, manuscript in preparation). As the fibrillar medin preparation may contain not only fibrils but also smaller aggregates of medin, we centrifuged $(25000 \mathrm{~g}$, $15 \mathrm{~min}$ ) such material and studied the effects of the supernatant. When fibrillar medin is centrifuged, a pellet that contains fibrils is formed (Congo-positive), whereas smaller aggregates end up in the supernatant. The medin solutions or an equivalent amount of dimethylsulfoxide were diluted directly into the medium for a final peptide concentration of $20 \mu \mathrm{mol} / \mathrm{l}$. At day 3 , the cell media were removed and mixed with $10 \%$ glycerol and stored at $-70^{\circ} \mathrm{C}$ for gelatin zymography and Western blot analyses. The cells were washed once with serum-free medium, and cell medium containing 10\% Alamar Blue (Sigma, St Louis, MO, USA) was added to the cells for $45 \mathrm{~min}$. Cell proliferation was determined at wavelengths of $530 \mathrm{~nm}$ excitation and $590 \mathrm{~nm}$ emission with a Wallac Victor ${ }^{2} 1420$ multilabel counter (Perkin Elmer, Turku, Finland). The result of the Alamar Blue assay was based on the analysis of triplicate wells in three independent experiments and correlated well with the number of cells estimated with a cell counter.

\section{Gelatin Zymography}

Cell medium was separated on $10 \%$ polyacrylamide gels containing $0.1 \%$ sodium dodecyl sulfate (SDS) and $0.1 \%$ gelatin. To ensure that an equivalent amount of protein was loaded in each well, the amount was correlated to the cell number. To retain protease activity, the samples were not heated with the sample buffer and the separation was run under moderate voltage $(80 \mathrm{~V})$. After electrophoresis, gels were agitated for $30 \mathrm{~min}$ in Tris-buffered saline (TBS) $(0.05 \mathrm{~mol} / \mathrm{l}$ Tris- $\mathrm{HCl}, 0.15 \mathrm{~mol} / \mathrm{l} \mathrm{NaCl}, \mathrm{pH} 7.4)$ containing $5 \mathrm{mmol} / \mathrm{l} \mathrm{CaCl} 2,5 \mu \mathrm{mol} / \mathrm{l} \mathrm{ZnCl}_{2}$ and $2.5 \%$ Triton X-100. Gels were further incubated for $40 \mathrm{~h}$ at $37^{\circ} \mathrm{C}$ in the same buffer as in the previous step but with $1 \%$ Triton X-100. During this step, gelatinases present in the gel are activated and cleave the gelatin in the gel. The gels were stained in Coomassie Blue solution until proteolytic activity was observed as white bands against a blue background.

\section{SDS-PAGE and Western Blotting}

Extracted proteins from patient tissue specimens and cell media were used for Western blotting. Cell media were mixed with equal amounts of sample buffer containing $8 \%$ SDS and $0.02 \mathrm{~mol} / \mathrm{l}$ dithiothreitol, boiled and separated by SDS-polyacrylamide gel electrophoresis (PAGE) using 10\% acryl/bisacrylamide gels. ${ }^{34}$ Extracted proteins from patient tissue specimens (dissolved in sample buffer containing 4\% SDS) were resolved according to Schägger and von $\operatorname{Jagow}^{34}$ on a $16.5 \%$ acryl/bis-acrylamide gel. The proteins were blotted to nitrocellulose Hybond ${ }^{\mathrm{TM}}$-ECL ${ }^{\mathrm{TM}}$ membranes (Amersham Biosciences, Uppsala, Sweden). The membrane containing the proteins from the cell media was incubated with a mousemonoclonal MMP-2 antibody (IM33T, Calbiochem, San Diego, CA, USA) diluted 1:500, and the membrane with patient-extracted proteins was incubated with medin antisera A179, diluted 1:2000.

\section{Statistical Methods}

Statistical analyses were performed with nonparametric (Spearman) correlation and the Mann-Whitney test. Values were given as mean \pm s.e.m. $P<0.05$ was considered significant.

\section{RESULTS \\ Histological Findings}

Aneurysm and dissection specimens showed varying degrees of aortic medial degeneration. Areas rich in alcian blue positive material were commonly found (Figure 1a and b), besides signs of fragmentation of the elastic lamina. Loss of smooth muscle nuclei was evident in some areas in many of the specimens.

A majority of the samples, including the control materials, contained amyloid in the extracellular matrix of the media. The deposits had the characteristics of medin-derived amyloid, ${ }^{11,35}$ with small widely spread aggregates. Amyloid was often, but not always, observed as thin bands closely connected to the elastic laminae (Figure $1 \mathrm{c}$ and d). Occasionally, the amyloid deposits occurred in close association with fragmented ends of elastic laminae. Small amyloid deposits, which were apparently located within smooth muscle cells, were also commonly seen (not shown).

All amyloid deposits labeled evenly with antiserum A179 against medin (Figures $1 \mathrm{c}$ and $\mathrm{d}$ and $2 \mathrm{a}-\mathrm{d}$ ). In addition, a more granular and often widely distributed immunolabeling was seen in areas without congophilic material (Figures 1e and $\mathrm{f}$ and $2 \mathrm{e}-\mathrm{h}$ ). Such labeling occurred occasionally in the control material but was much more conspicuous in aortic specimens from aneurysms and dissections.

\section{Medin Amyloid in the Three Different Groups}

The amount of amyloid (Figure 3), measured as the number of congophilic particles per area, was significantly larger in the control group $(2.37 \pm 0.58$; median $=1.20)$ compared to the aneurysm $(0.63 \pm 0.13, P=0.03$; median $=0.42)$ and dissection groups $(0.36 \pm 0.24, P=0.01 ; \quad \operatorname{median}=0.02)$. There was a significant correlation between the degree of medin-derived amyloid and patient age when all the aortic specimens were evaluated together, $r=0.65 P<0.0001$ (data 

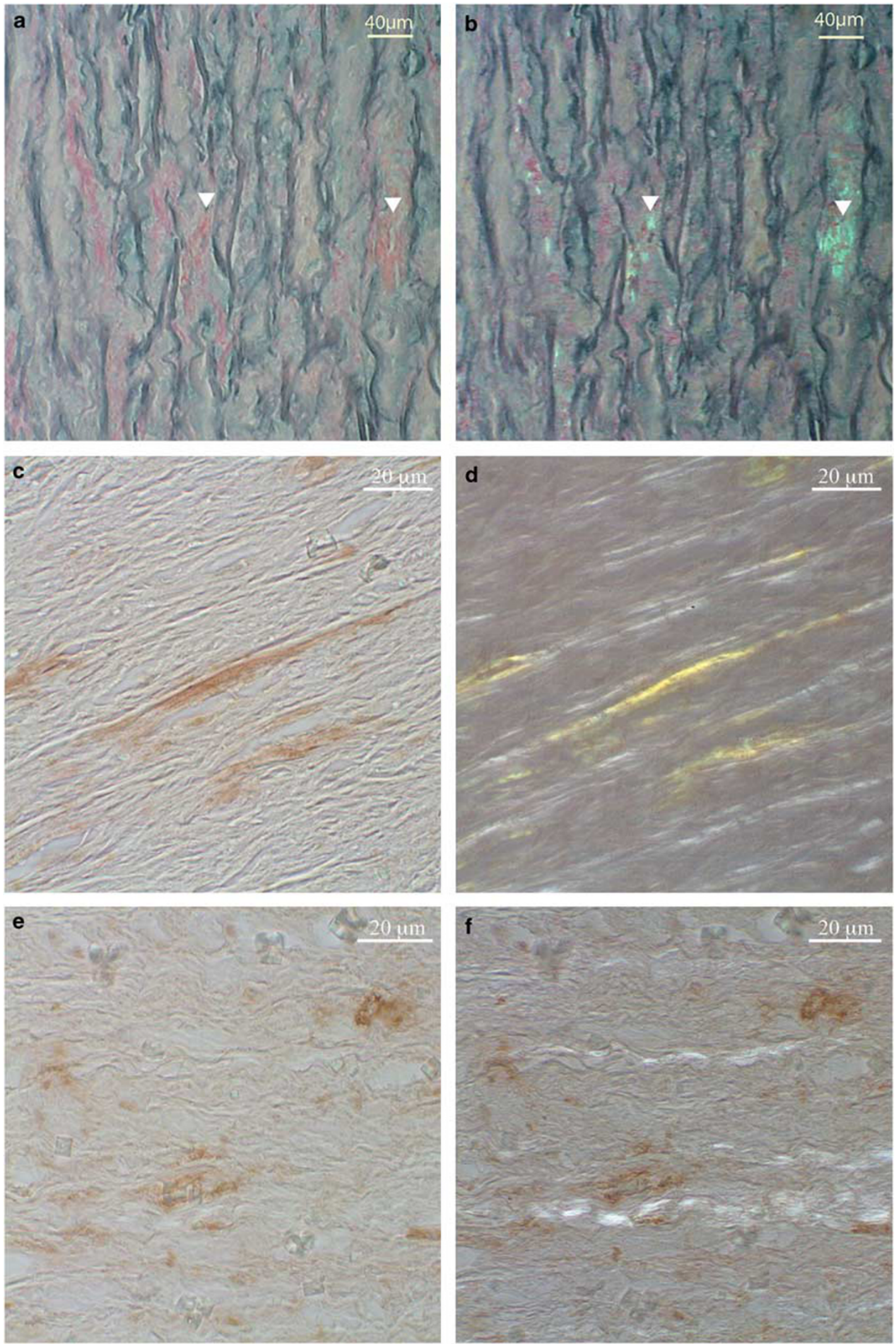

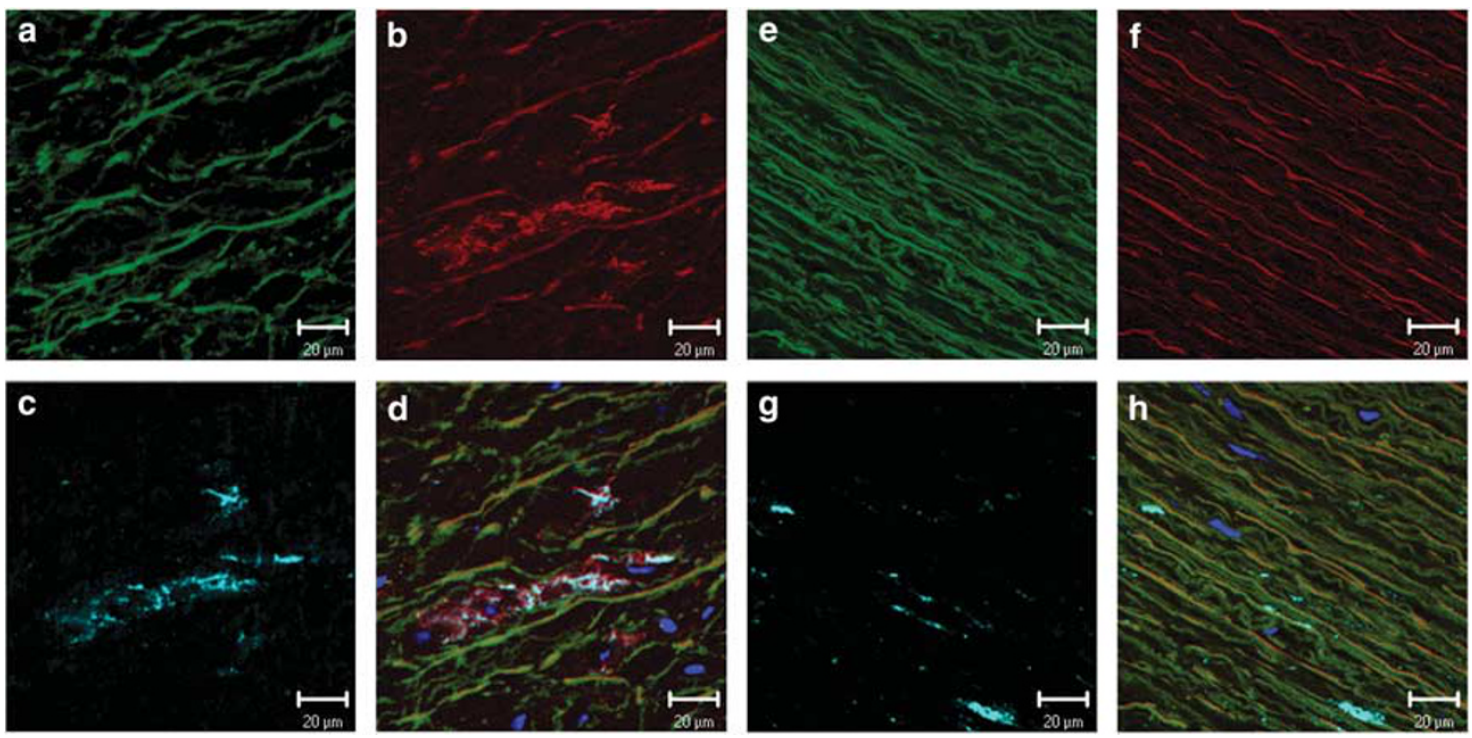

Figure 2 Confocal microscopy of a typical control (a-d) and aneurysm (e-h) specimen. The sections were stained with Congo red and labeled with medin antiserum A179. Elastic fibers give rise to auto-fluorescence and are green (a and $\mathbf{e}$ ). The red color shows the amyloid aggregates stained with Congo red. The elastic fibers are also auto-fluorescent at this wavelength. Only the control specimen (b), not the aneurysm (f), displays amyloid. Immunostaining with the medin antiserum (light blue) is seen in figure $\mathbf{c}$ and $\mathbf{g}$. A fourth wavelength was used to detect Hoechst-stained nuclei of smooth muscle cells (dark blue), but is not shown separately. Figures $\mathbf{d}$ and $\mathbf{h}$ reflect the merged images of all 4 wavelengths. In the control specimen, the antiserum only labels the amyloid (c and $\mathbf{d}$ ). In the aneurysm specimen, a granular immunolabeling but no amyloid is observed ( $\mathbf{g}$ and $\mathbf{h}$ ), indicating the presence of small medin aggregates.

not shown). It should also be noted that the percentage of specimens lacking any amyloid deposits was almost identical in the control and aneurysm materials (21 and 22\%, respectively). The number of specimens in the dissection group was too small for comparison.

\section{Medin Immunoreactivity}

In the control specimens, medin immunoreactivity was seen mainly in association with congophilic deposits. In accordance with this, there was a strong correlation between the amount of amyloid and the medin immunoreactive area in the control specimens $(r=0.91, P<0.0001)$ (Figure 4a). This correlation was not found in the materials from aortic aneurysms or dissections $(r=0.08$, NS and $r=0.27$, NS, respectively; Figure $4 \mathrm{~b}$ and $\mathrm{c}$ ). The medin immunoreactive areas did not differ significantly between the three different groups, although there was a tendency for a higher value in the aneurysm group $(6.02 \pm 0.79$; median $=4.42)$ compared to the dissection $(4.92 \pm 0.67$; median $=4.50)$ and control groups $(4.54 \pm 0.74$; median $=4.37) \quad($ Figure $4 \mathrm{~d})$. These results show that the aneurysm and dissection groups contain more medin immunoreactivity not associated with amyloid aggregates and may indicate the presence of medin oligomers.

\section{Extracted Proteins}

After homogenization and centrifugation of tissues containing amyloid, which is water-insoluble, the amyloid fibrils end up in the pellet. Amyloid specimens separated by SDS-PAGE often give rise to a characteristic smear also containing several immunoreactive bands of different sizes. These bands represent monomers and polymers of the amyloid peptide. In Western blot analyses of proteins extracted from pellet material of amyloid-containing specimens, an immunoreactive band of medin size $(6 \mathrm{kDa})$ was seen (not shown). In addition, multiple immunoreactive bands of larger size, including

Figure 1 Histological findings. (a) and (b) A section from a patient with thoracic aortic aneurysm stained with alcian blue for mucoid substance (blue) and with Congo red for amyloid (arrow heads). In ordinary light (a) Congo-stained amyloid appears red and in polarized light (b) amyloid acquires a typical green birefringence. (c) and (d) A section derived from a control specimen, which was double-stained for medin with antiserum A179 and Congo red. In normal light (c) the antibody labeling is visible and in polarized light (d) the characteristic yellow-green birefringence appears. As can be seen, the antibody labels the amyloid evenly and the amyloid is found along the elastic laminae. Within the control group, the antibody and Congo red stain usually co-localized and displayed the same pattern. (e) and (f) Aneurysm and dissection specimens usually contained medin immunoreactivity in areas where no amyloid was found. Medin immunoreactivity without the presence of amyloid is demonstrated in aortic material from an individual with aneurysm that was doublestained with A179 and Congo red stain (e). No green birefringence and consequently no amyloid is seen with polarized light (f). 
a band with mobility of lactadherin as well as a diffuse smear, were evident. Also water-soluble supernatants of homogenized materials from aneurysm, dissection and control specimens without amyloid were separated with SDS-PAGE and analyzed with Western blot (Figure 5). The results correlated well with the immunohistochemical data. Interest-

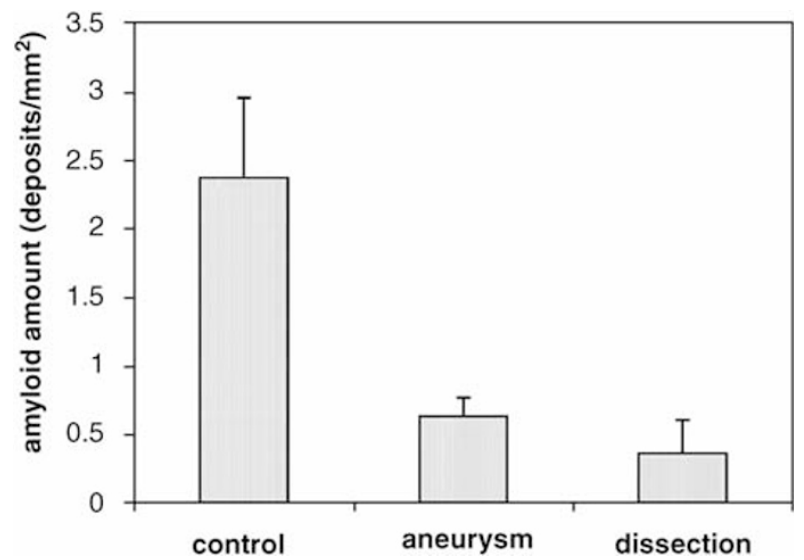

Figure 3 Medin amyloid in the three different groups. The amount of amyloid is shown as the mean \pm s.e.m. The degree of amyloid was significantly larger in the control group $(2.37 \pm 0.58)$ compared to the aneurysm $(0.63 \pm 0.13, P=0.03)$ and dissection $(0.36 \pm 0.24, P=0.01)$ groups. ingly, the aneurysm and dissection material showed strong immunoreaction. A band around $46 \mathrm{kDa}$, corresponding to the size of lactadherin as well as a typical amyloid smear

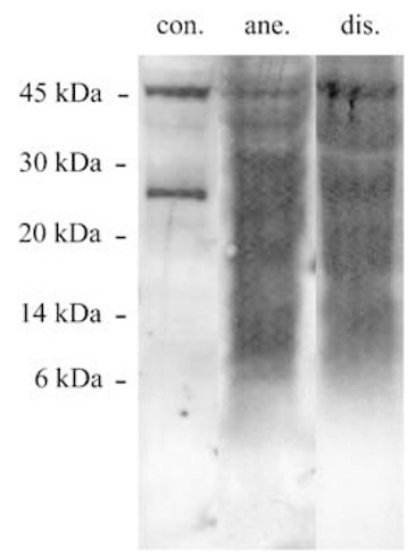

Figure 5 Western blot with medin antiserum A179 on water-soluble homogenized material (supernatants) from a control (left lane), an aneurysm (middle lane) and a dissection (right lane) specimen. The three specimens contained no amyloid. The aneurysm and dissection show stronger immunoreactivity than the control specimen, which only displays lactadherin immunoreactivity at 46 and $26 \mathrm{kDa}$. In addition, the two disease specimens display typical amyloid smears from $6 \mathrm{kDa}$ (monomeric medin) and upward, probably representing different aggregative states of medin and indicating the presence of oligomers. a

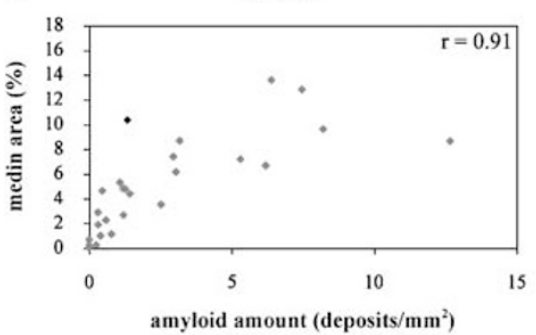

b

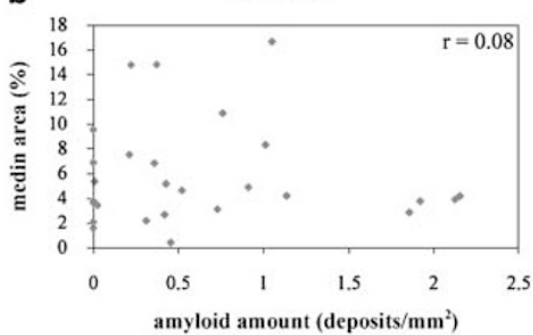

c

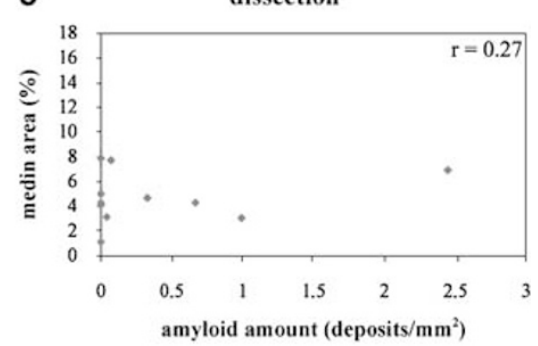

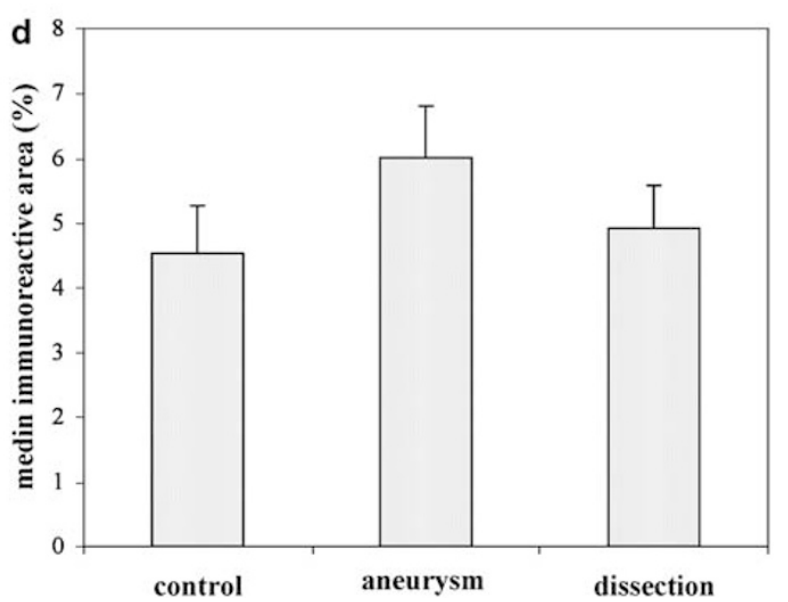

Figure 4 (a-c) The relationship between the amount of amyloid (congophilic deposits) and the area taken up by medin immunoreactivity in the three studied groups. There is a strong correlation $(r=0.91, P<0.0001)$ between the two variables for the control material, but not for the two diseased groups. (d) Medin immunoreactive area (\%) in the thoracic aortic media in the three studied groups. There is no significant difference between the groups. 
down to $6 \mathrm{kDa}$, which is the size of monomeric medin, was seen. The control material contained the $46 \mathrm{kDa}$ lactadherin, as expected, but no smear. A band around $26 \mathrm{kDa}$ was also evident in the control material and probably represents a truncated form of lactadherin, which has been described earlier by Cavaletto et $a l^{36}$ in the lactating gland. As fibrillar amyloid proteins end up in the pellet material, these watersoluble medin aggregates probably represent smaller medin aggregates, thus further supporting the presence of oligomers. The immunoreaction was not seen when antiserum absorbed with the unconjugated peptide was used.

\section{Cell Studies and Toxicity Studies}

Medin, which is a 50 amino-acid residue long peptide, aggregates spontaneously to form amyloid-like fibrils when incubated in vitro at the experimental conditions used here. This aggregation typically takes 5 days and is preceded by the formation of oligomeric assemblies. We examined the effect of medin in vitro by adding the peptide of different aggregative states to aortic smooth muscle cells in culture. Figure 6a shows the percentage of viable cells for the different medin categories tested with the Alamar Blue assay (resazurin). This assay is a sensitive and simple method for the measurement of cell viability. The reagent is added to the cell medium in an oxidized form and is then reduced to a fluorescent dye by cell activity. ${ }^{37}$ Cells treated with fibrillar and centrifuged medin do not grow as well as the control. The percentage of viable cells is only $88 \pm 2.93 \%, P<0.05$ for the fibrillar medin and $89 \pm 3.09 \%, P<0.05$ for the centrifuged medin compared to the control. No toxic effect is seen for the cells treated with freshly dissolved medin, $101 \pm 2.53 \%$, NS. Because fibrillar medin and the supernatant of fibrillar medin show the same deleterious effect, the mature fibrils are probably not the toxic species. It is more likely that some smaller aggregates of medin (i.e. oligomers) exert the toxic effect observed in the cell culture experiments.

Cell media were collected and electrophoresed on polyacrylamide gels containing gelatin. Cells treated with medin showed an increased gelatinase activity compared to cells exposed to media lacking medin or media containing control (nonrelevant) peptides. Gelatinases with molecular weights between 66 and $80 \mathrm{kDa}$ were especially upregulated by medin (Figure 6b). No expression of gelatinases was detected in cell medium coincubated with medin without cells, demonstrating that the gelatinase activity is cell mediated as expected (data not shown).

With the use of an antibody directed against MMP-2 (Figure 6c) in Western blot, we showed that MMP-2 is partly responsible for the upregulation of gelatinases seen with the zymography. MMP-2 is a protease that degrades both collagen and elastin and has a size of $72 \mathrm{kDa}$ in its inactive state. During activation, a pro-peptide segment is removed and a $66 \mathrm{kDa}$ protein remains. Both the inactive and active forms of MMP-2 were induced in medin-treated cells compared to

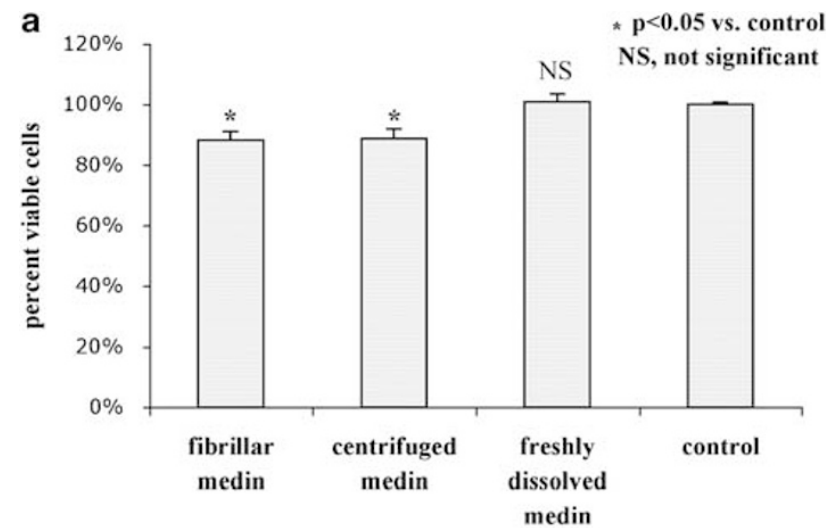

b

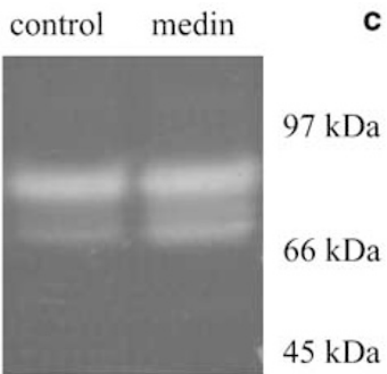

control medin

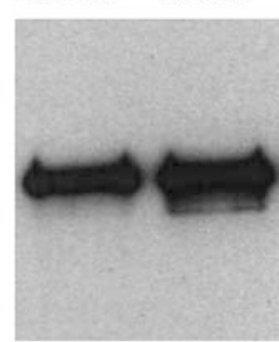

Figure 6 Medin in in vitro studies. (a) Aortic smooth muscle cells were coincubated with medin that was fibrillar (positive with Congo red), freshly dissolved (mainly monomers) or the supernatant of the centrifuged fibrillar medin preparation. The centrifugation of fibrillar medin results in a pellet, which mainly consists of mature fibrils. The supernatant contains aggregated medin that has not yet formed fibrils. Cells in the presence of fibrillar and centrifuged medin showed the same toxic response, and only 88 and $89 \%$, respectively, of the cells were viable compared to the control. Cells treated with freshly dissolved medin appeared non-affected. (b) Culture media from cells incubated with medin showed an increased gelatinase activity in a gelatin zymography assay. An induction of proteases in the size range of $66-80 \mathrm{kDa}$ was seen. (c) Matrix metalloproteinase 2, which is a protein that degrades elastin and collagen, is one of the proteases upregulated by medin. Both the inactive $(72 \mathrm{kDa})$ and the active (66 kDa) forms of MMP-2 are induced.

control cells. Similar results were observed with smooth muscle cells of uterine origin (data not shown).

\section{DISCUSSION}

Medin (AMed) amyloid is found in a majority of individuals above the age of 50 years. Small, but sometimes abundant, deposits are seen within the aortic media, usually in close contact with the elastic laminae. ${ }^{9,38}$ No specific disease has been attributed to medin amyloid deposits. However, as it has been shown that amyloids of other biochemical nature found in the vasculature may cause a weakening of the vessel wall, ${ }^{27-29}$ it is reasonable to believe that medin is deleterious to the surrounding tissue and is also involved in conditions such as thoracic aortic dissections and aneurysms.

The observed effects of medin in an aortic smooth muscle cell culture indicate that medin amyloid may be an important player in the development of aortic aneurysms and dissections by two separate pathways. First, cells exposed to medin 
express increased amounts of gelatinases, for example MMP-2. Thoracic aortic dissection and aneurysm often showed signs of fragmented elastin, which occasionally was surrounded by medin amyloid. It is possible that medin induces the production of MMP-2, which degrades the elastic laminae. The MMP gene expression has earlier been reported to be increased in patients suffering from thoracic aortic aneurysms or dissections. ${ }^{3-6}$ The effects of amyloid fibrils or oligomers on cells have been suggested to be of a generic nature, that is, it has been implied that it is the type of aggregation and not the biochemical nature of the peptide that determines the cellular response. It is therefore of interest that other studies have shown that $\mathrm{A} \beta$, the amyloidogenic peptide in Alzheimer's disease, induces the production of MMPs, ${ }^{39-41}$ which have been suggested to be of relevance for the development of intracerebral hemorrhage in patients with cerebral amyloid angiopathy.

Second, medin may be involved in the pathogenesis of thoracic aortic aneurysm and dissection by being toxic to the surrounding smooth muscle cells. Decreased cell viability was observed for aortic smooth muscle cells in the presence of oligomeric medin. Monomeric medin did not affect the cells and our results also indicate that mature fibrils are not toxic. The moderate toxicity that we saw in our experiment may be explained by the fact that the toxic oligomers are intermediate species, which are very short-lived in vitro and hard to capture during an experiment as the oligomers tend to aggregate further into fibrils. Thus, these results fit the emerging theory that specific aggregated proteins with an amyloid fold, probably in oligomeric forms rather than as fully developed amyloid fibrils, are involved in the pathogenesis of several degenerative disorders. ${ }^{21,22}$ Given this it has been proposed that mature amyloid may protect tissues from more deleterious amyloid oligomers. ${ }^{42}$ Although the target cells vary between the diseases, similar effects seem to be obtained irrespective of the biochemical nature of protein oligomers. One effect of amyloid oligomers is cell death through apoptosis. Why apoptosis occurs is still debated, but in vitro studies indicate that it may involve the formation of cell membrane pores ${ }^{23}$ or the generation of free radicals. ${ }^{24}$ In vivo increased oxidative stress ${ }^{43}$ and the presence of advanced glycation end products ${ }^{44,45}$ have been observed in amyloid-rich tissues from patients with different kinds of amyloidoses.

The finding of significantly less amyloid in the two investigation groups, as compared with the control material, may seem surprising. However, the medin immunoreactivity area did not differ between the three different groups. It is possible to speculate that aneurysms and dissections develop when medin fibrillogenesis proceeds relatively slowly, leading to the persistence of the more toxic oligomeric aggregates. The strong correlation between amyloid amount and medin immunoreactive area in the control material is explained by the finding that medin immunoreactivity almost exclusively occurred in the form of mature amyloid in those biopsies. The lack of significant correlation between the amount of amyloid and immunolabeled areas in both the aneurysm and dissection specimens depends on the sometimes pronounced granular non-amyloid immunoreactivity in the media. Although not definitely proved by our studies, it is probable that such substance is prefibrillar, possibly oligomeric and more water-soluble and may be the medin immunoreactive protein found with Western blot in the water extracts of amyloid-free aortic tissue.

Aging is a major risk factor for the development of any kind of amyloidosis and thereby also diseases associated with amyloid deposits. The reasons for this association are not known but may be multiple and include the increasing inability of the cells to efficiently handle misfolded and aggregation-prone protein species. In this study, we found a strong correlation between the amount of amyloid and age in the patient material.

In conclusion, we found significantly more medin-immunoreactive material not associated with amyloid in specimens from thoracic aortic aneurysms and dissections, which we interpreted as being medin oligomers. In addition, we show that small aggregates of medin, as opposed to fibrillar and monomeric medin, are toxic to aortic smooth muscle cells in vitro. Further, we found that medin induces smooth muscle cell production of MMP-2 and other gelatinases that may degrade the elastic laminae and collagen of the aorta. Medin may thus weaken the aortic media in two ways, first by killing the smooth muscle cells and second by inducing the degradation of elastin and collagen. The toxicity mechanism of medin is still unresolved and should be explored further, both in vitro and in vivo. Other studies with the aim to identify therapeutic agents that interfere with the toxic species are of great interest. In a recent study, the last 19 amino-acid residues of the medin peptide were found to be required for amyloid formation. ${ }^{46}$ One therapeutic approach would be to find agents that interact with this motif, thereby inhibiting the generation of medin oligomers and preventing toxicity.

\section{ACKNOWLEDGEMENT}

We thank Gunilla T Westermark for advice and Dr Kenneth $\mathrm{H}$ Johnson for commenting on this manuscript. This study was supported by the Swedish Research Council and the Swedish Heart-Lung Association.

\section{DISCLOSURE/DUALITY OF INTEREST}

The authors state no duality of interest.

1. Klima T, Spjut HJ, Coelho A, et al. The morphology of ascending aortic aneurysms. Hum Pathol 1983;14:810-817.

2. DeSanctis RW, Doroghazi RM, Austen WG, et al. Aortic dissection. N Engl J Med 1987;317:1060-1067.

3. Koullias GJ, Ravichandran P, Korkolis DP, et al. Increased tissue microarray matrix metalloproteinase expression favors proteolysis in thoracic aortic aneurysms and dissections. Ann Thorac Surg 2004;78:2106-2110.

4. Taketani $T$, Imai $Y$, Morota $T$, et al. Altered patterns of gene expression specific to thoracic aortic aneurysms: microarray analysis of surgically resected specimens. Int Heart J 2005;46:265-277.

5. Lesauskaite V, Tanganelli $P$, Sassi $C$, et al. Smooth muscle cells of the media in the dilatative pathology of ascending thoracic aorta: 
morphology, immunoreactivity for osteopontin, matrix metalloproteinases, and their inhibitors. Hum Pathol 2001;32: 1003-1011.

6. Barbour JR, Stroud RE, Lowry AS, et al. Temporal disparity in the induction of matrix metalloproteinases and tissue inhibitors of metalloproteinases after thoracic aortic aneurysm formation. J Thorac Cardiovasc Surg 2006;132:788-795.

7. Schwartz P. Cardiovascular amyloidosis. In: Amyloidosis, Cause and Manifestation of Senile Detoriation. Charles C Thomas: Springfield, 1970, p 80-110.

8. Iwata T, Kamei T, Uchino F, et al. Pathological study on amyloidosisrelationship of amyloid deposits in the aorta to aging. Acta Pathol Jpn 1978;28:193-203.

9. Mucchiano G, Cornwell III GG, Westermark P. Senile aortic amyloid. Evidence for two distinct forms of localized deposits. Am J Pathol 1992;140:871-877.

10. Cornwell III GG, Murdoch WL, Kyle RA, et al. Frequency and distribution of senile cardiovascular amyloid. A clinicopathologic correlation. Am J Med 1983;75:618-623.

11. Häggqvist B, Näslund J, Sletten $K$, et al. Medin: an integral fragment of aortic smooth muscle cell-produced lactadherin forms the most common human amyloid. Proc Natl Acad Sci USA 1999;96:8669-8674.

12. Ceriani RL, Sasaki M, Sussman $H$, et al. Circulating human mammary epithelial antigens in breast cancer. Proc Natl Acad Sci USA 1982;79:5420-5424.

13. Hanayama R, Tanaka M, Miwa K, et al. Identification of a factor that links apoptotic cells to phagocytes. Nature 2002;417:182-187.

14. Carmon L, Bobilev-Priel I, Brenner B, et al. Characterization of nove breast carcinoma-associated BA46-derived peptides in HLA-A2.1/D(b)beta2m transgenic mice. J Clin Invest 2002;110:453-462.

15. Hamosh M, Peterson JA, Henderson TR, et al. Protective function of human milk: the milk fat globule. Semin Perinatol 1999;23:242-249.

16. Shi J, Gilbert GE. Lactadherin inhibits enzyme complexes of blood coagulation by competing for phospholipid-binding sites. Blood 2003;101:2628-2636

17. Silvestre JS, Thery C, Hamard G, et al. Lactadherin promotes VEGFdependent neovascularization. Nat Med 2005;11:499-506.

18. Larsson A, Peng $\mathrm{S}$, Persson $\mathrm{H}$, et al. Lactadherin binds to elastin-a starting point for medin amyloid formation? Amyloid 2006;13:78-85.

19. Merlini G, Bellotti V. Molecular mechanisms of amyloidosis. N Engl J Med 2003;349:583-596.

20. Westermark P. Aspects on human amyloid forms and their fibril polypeptides. FEBSJ 2005;272:5942-5949.

21. Bucciantini M, Giannoni $\mathrm{E}$, Chiti F, et al. Inherent toxicity of aggregates implies a common mechanism for protein misfolding diseases. Nature 2002;416:507-511.

22. Kayed R, Head E, Thompson JL, et al. Common structure of soluble amyloid oligomers implies common mechanism of pathogenesis. Science 2003;300:486-489.

23. Thundimadathil J, Roeske RW, Jiang HY, et al. Aggregation and porinlike channel activity of a beta sheet peptide. Biochemistry 2005;44:10259-10270.

24. Schubert D, Behl C, Lesley $R$, et al. Amyloid peptides are toxic via a common oxidative mechanism. Proc Natl Acad Sci USA 1995;92: 1989-1993.

25. Behl C, Davis JB, Lesley $\mathrm{R}$, et al. Hydrogen peroxide mediates amyloid beta protein toxicity. Cell 1994;77:817-827.

26. Sousa MM, Du Yan $S$, Fernandes $R$, et al. Familial amyloid polyneuropathy: receptor for advanced glycation end productsdependent triggering of neuronal inflammatory and apoptotic pathways. J Neurosci 2001;21:7576-7586.
27. Revesz T, Ghiso J, Lashley T, et al. Cerebral amyloid angiopathies: a pathologic, biochemical, and genetic view. J Neuropathol Exp Neurol 2003:62:885-898.

28. Levy E, Carman MD, Fernandez-Madrid IJ, et al. Mutation of the Alzheimer's disease amyloid gene in hereditary cerebral hemorrhage, Dutch type. Science 1990;248:1124-1126.

29. Winkler DT, Bondolfi L, Herzig MC, et al. Spontaneous hemorrhagic stroke in a mouse model of cerebral amyloid angiopathy. J Neurosci 2001;21:1619-1627.

30. Christie R, Yamada M, Moskowitz $M$, et al. Structural and functional disruption of vascular smooth muscle cells in a transgenic mouse model of amyloid angiopathy. Am J Pathol 2001;158:1065-1071.

31. Puchtler $H$, Sweat $F$, Levine $M$. On the binding of Congo red by amyloid. J Histochem Cytochem 1962;10:355-364.

32. Westermark GT, Johnson KH, Westermark P. Staining methods for identification of amyloid in tissue. Methods Enzymol 1999;309:3-25.

33. Gustavsson $\AA$, Engström U, Westermark P. Mechanisms of transthyretin amyloidogenesis. Antigenic mapping of transthyretin purified from plasma and amyloid fibrils and within in situ tissue localizations. Am J Pathol 1994;144:1301-1311.

34. Schägger $H$, von Jagow $G$. Tricine-sodium dodecyl sulfatepolyacrylamide gel electrophoresis for the separation of proteins in the range from 1 to $100 \mathrm{kDa}$. Anal Biochem 1987;166:368-379.

35. Peng S, Glennert J, Westermark P. Medin-amyloid: A recently characterized age-associated arterial amyloid form affects mainly arteries in the upper part of the body. Amyloid 2005;12:96-102.

36. Cavaletto M, Giuffrida MG, Giunta C, et al. Multiple forms of lactadherin (breast antigen BA46) and butyrophilin are secreted into human milk as major components of milk fat globule membrane. J Dairy Res 1999;66:295-301.

37. O'Brien J, Wilson I, Orton T, et al. Investigation of the Alamar Blue (resazurin) fluorescent dye for the assessment of mammalian cell cytotoxicity. Eur J Biochem 2000;267:5421-5426.

38. Peng S, Westermark GT, Näslund J, et al. Medin and medin-amyloid in ageing inflamed and non-inflamed temporal arteries. J Pathol 2002;196:91-96

39. Deb S, Wenjun Zhang J, Gottschall PE. Beta-amyloid induces the production of active, matrix-degrading proteases in cultured rat astrocytes. Brain Res 2003;970:205-213.

40. Jung SS, Zhang W, Van Nostrand WE. Pathogenic A beta induces the expression and activation of matrix metalloproteinase-2 in human cerebrovascular smooth muscle cells. J Neurochem 2003;85: 1208-1215.

41. Lee JM, Yin KJ, Hsin I, et al. Matrix metalloproteinase-9 and spontaneous hemorrhage in an animal model of cerebral amyloid angiopathy. Ann Neurol 2003;54:379-382.

42. Caughey B, Lansbury PT. Protofibrils, pores, fibrils, and neurodegeneration: separating the responsible protein aggregates from the innocent bystanders. Annu Rev Neurosci 2003;26:267-298.

43. Ando $\mathrm{Y}$, Nyhlin $\mathrm{N}$, Suhr $\mathrm{O}$, et al. Oxidative stress is found in amyloid deposits in systemic amyloidosis. Biochem Biophys Res Commun 1997;232:497-502.

44. Vitek MP, Bhattacharya K, Glendening JM, et al. Advanced glycation end products contribute to amyloidosis in Alzheimer disease. Proc Natl Acad Sci USA 1994;91:4766-4770.

45. Nyhlin N, Ando $\mathrm{Y}$, Nagai $\mathrm{R}$, et al. Advanced glycation end product in familial amyloidotic polyneuropathy (FAP). J Intern Med 2000;247: 485-492.

46. Larsson A, Söderberg L, Westermark GT, et al. Unwinding fibril formation of medin, the peptide of the most common form of human amyloid. Biochem Biophys Res Commun 2007;361:822-828. 